\title{
ESTIMATION OF SPERMATOZOA NUMBERS USING A MICROPORE FILTRATION TECHNIQUE
}

\author{
E. F. NUTTING \\ Department of Endocrinology, Division of Biological Research, \\ G. D. Searle $\Xi$ Co., Chicago, Ill., U.S.A.
}

(Received 14th May 1969)

The estimation of sperm concentration of semen samples has been accomplished by a variety of different methods. The equipment used in these techniques has ranged from the simple haemocytometer chamber to more highly sophisticated electronic means such as measuring light transmission (Willet \& Buckner, 1951) and counting particle size (Glover \& Phipps, 1962). Although electronic means of counting spermatozoa offer obvious advantages in situations where large numbers of routine counts are involved, estimates made with a haemocytometer have been the method of choice for most researchers because the technique is relatively accurate, utilizes simple inexpensive equipment and is rapid. In addition, the haemocytometer, unlike electronic methods, has been used successfully in conjunction with centrifugation to study the movement of spermatozoa through the female reproductive tract (Braden, 1953). This method, however, was not entirely satisfactory in our laboratory when it was applied to reproductive studies involving pharmacological means of impeding sperm migration because the total number of spermatozoa contained in oviducal washings from treated animals was frequently reduced to only a few spermatozoa or none at all.

In an attempt to develop a more suitable method to use under these conditions, micropore filtration techniques that had been developed for the cytological study of cells in body fluids and the washings of various organs, were modified to count spermatozoa. In the sequence of experiments to be described, semen samples were used to establish the feasibility of the method, which was applied to the recovery of spermatozoa from the female reproductive tract.

Rabbit semen was collected with the aid of an artificial vagina and ejaculates from several males were pooled to provide the samples for sperm counts. Samples were diluted with $0.9 \% \mathrm{NaCl}$ solution and $0.1 \mathrm{ml}$ of diluted semen was filtered. The filtration unit consisted of a 5-ml hypodermic syringe attached to a Swinny filter holder containing a 13-mm type $\mathrm{HA}$ filter with pore size of $0.45 \pm 0.2 \mu$. The filter was moistened with physiological saline $(0.9 \% \mathrm{NaCl}$ solution in distilled water) before use. Organ washings were obtained from adult oestrous rabbits (Dutch belted strain) that were inseminated artificially with $0.5 \mathrm{ml}$ of semen diluted with physiological saline to a concentration of $20 \times 10^{6}$ spermatozoa/ml. Animals were killed $16 \mathrm{hr}$ after insemination. Physiological saline $(2 \mathrm{ml}$ ) was forced through one end of each uterine horn or 
oviduct. The washing fluid emerging from the opposite end of the organ was collected into a 5-ml hypodermic syringe attached to the filtration unit described above. The plunger was replaced and the washing fluid was forced through the filter.

After filtration of the sample, 5 to $10 \mathrm{ml}$ of saline were forced through the filter to remove as much water-soluble detritus from the surface as possible. Next, 2 to $3 \mathrm{ml}$ of $70 \%$ ethanol were passed through the filter to fix the spermatozoa and exfoliative cells adhering to the surface. Filters were then either stored temporarily in $95 \%$ ethanol or stained immediately with haematoxylin and eosin and cleared in xylol using the method described by the Millipore Filter Corporation (ADM-50 Techniques for exfoliative cytology.) The filters were mounted on slides and cover slips were attached using an excess of an acrylic mounting medium. Slides were usually viewed immediately with a microscope $(\times 400)$ equipped with a calibrated eyepiece reticle. A pre-arranged sequence of seven fields was scanned and the number of spermatozoa counted. The total number of spermatozoa on the surface of the membrane was estimated by the following calculation: estimated number of spermatozoa $=$ (number of spermatozoa counted $\times$ effective filtration area $) \div(7 \times$ area of microscopic field $)$. The area of the membrane and the area of the microscopic field were constants with values of 78.54 and $0.062 \mathrm{~mm}^{2}$, respectively.

For the haemocytometer method, three chambers (Hausser chamber with double Neubauer ruling) were filled from a single aliquot of the diluted sample of semen. The cells were allowed to settle and five large squares $\left(0.02 \mathrm{~mm}^{3}\right)$ in each chamber were counted under the microscope $(\times 400)$. The final estimate of sperm concentration was based on the average counts of the three chambers.

In the first experiments, each of four pooled ejaculates of semen was serially diluted to concentrations of $10 \%, 1 \%$ and $0.1 \%$ and sperm counts were made on duplicate samples to determine the optimum concentration for counting with a micropore filter. With a dilution of $10 \%$, sperm density was too great to count accurately. The estimates of sperm concentrations for the $1 \%$ and $0.1 \%$ semen dilutions were compared by an analysis of variance. Estimates obtained with a concentration of $0.1 \%$ were significantly $(P<0.01)$ larger $\left(329.4 \times 10^{6} /\right.$ $\mathrm{ml}$ ) than with a dilution of $1 \%\left(79.55 \times 10^{6} / \mathrm{ml}\right)$. Evidently, the density of spermatozoa was still too great with the latter concentration to obtain accurate counts. Thus, $0.1 \%$ appeared to be about the optimum semen concentration and was used in subsequent testing.

Next, the optimum concentration of semen for counting with the haemocytometer was determined. Three separate aliquots from a sample of pooled semen were each serially diluted to concentrations of 10,1 and $0.1 \%$. Each of the nine diluted samples was counted by three readers. The results were evaluated using an analysis of variance. Differences in estimates obtained from different readers were significant $(P<0.05)$. Average estimates of sperm number obtained with dilutions of $10 \%\left(197.8 \times 10^{6} / \mathrm{ml}\right)$ and $0.1 \%\left(218.7 \times 10^{6} / \mathrm{ml}\right)$ were consistently lower than with a semen dilution of $1 \%\left(313.9 \times 10^{6} / \mathrm{ml}\right)$. Thus, a dilution of $1 \%$ semen appeared to be optimal for estimation of sperm numbers with the haemocytometer chamber.

In the third experiment, the two methods of counting were compared directly. 
The sperm numbers in eighteen different semen samples of pooled ejaculates, each collected from three to five rabbits, were estimated from counts obtained with a haemocytometer chamber and a micropore filter using the optimal dilutions of $1 \%$ and $0.1 \%$, respectively, determined in the first two experiments. Text-figure 1 is a scatter diagram of the micropore filter counts plotted against the corresponding haemocytometer counts. The correlation coefficient was $0 \cdot 85$. The mean values of all the haemocytometer counts and micropore filter counts were $460 \times 10^{6}$ and $399 \times 10^{6}$ spermatozoa, respectively. The difference between the means was not significant. Thus, counts obtained with the micropore filter technique yielded estimates of sperm numbers that closely approximated to those obtained with the haemocytometer chamber.

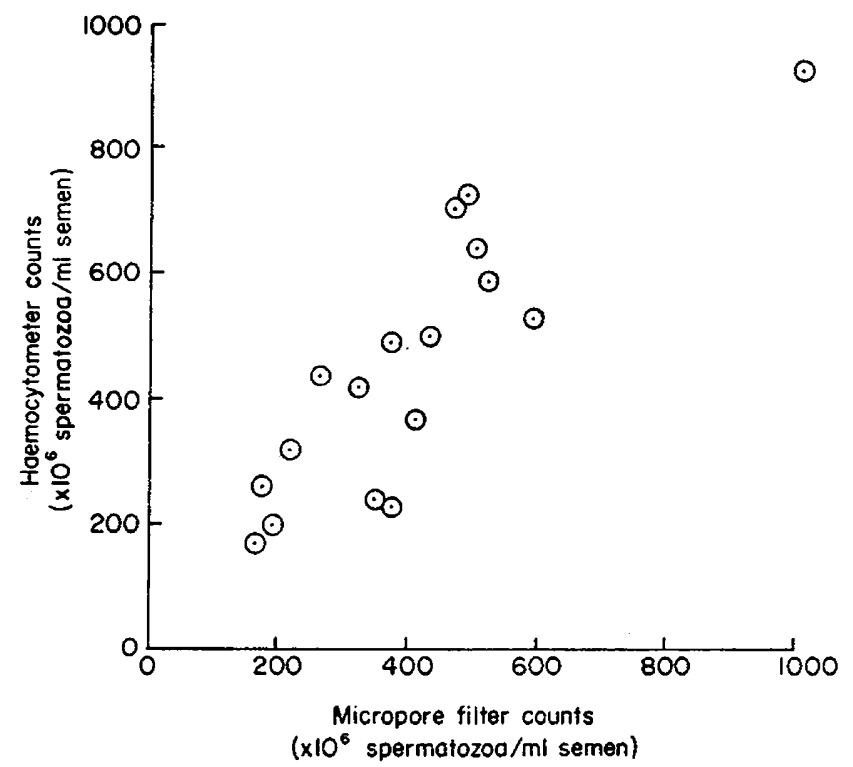

TExT-FIG. 1. Scatter diagram showing the correlation between sperm counts estimated with the haemocytometer chamber and with the micropore filtration method. Each point represents the mean number of spermatozoa of three separate determinations from the same semen sample by each method.

The time required to make a single count by this method, using a total of three filters, averaged about $45 \mathrm{~min}$. However, we have since found that all steps associated with the staining of the preparation can be eliminated when a phase contrast microscope is used for the counting of spermatozoa. In this case, the fixed filter was dipped successively into propanol for $2 \mathrm{~min}$, propanol plus xylol ( $1: 1)$ for 2 min and xylol for 5 or more min. Counts were made immediately following mounting in xylol or acrylic mounting media. When the procedure was modified in this manner, the total length of time for preparation of the sample and routine counting of three filters was reduced to approximately 20 min. Counts made with the haemocytometer chamber required about the same length of time.

The ranges of the estimates of the number of spermatozoa recovered from the 
washings from the uterus and oviducts of four rabbits by the micropore filtration technique were $69 \times 10^{3}$ to $274 \times 10^{3}$ and 4668 to 8796 , respectively. Although the number of animals was small, the average number of spermatozoa recovered agreed reasonably well with estimates made by other methods (Austin 1948; Chang, 1951; Braden, 1953). The spermatozoa from the fluid were concentrated on the filter and appeared to be evenly distributed as a monolayer across the surface. However, in some instances epithelial cells, leucocytes and detritus, contained in the wash fluid, accumulated on the filter surface and interfered with the accuracy of the count. An attempt to reduce contaminants by pre-filtration of the sample through filters with a pore size diameter of $8 \mu$ was unsuccessful due to retention of large numbers of spermatozoa on the filter surface. In some cases, visualization of spermatozoa was difficult because the filter and/or materials on the surface also took up the stain. However, in most instances, satisfactory visualization was attained with a higher intensity of illumination. In a few samples taken from pseudopregnant rabbits, the high concentration of exfoliative cells and surface detritus prevented accurate counting. In studies where conditions such as this were likely to be encountered, the use of negative pressure and filters with greater surface area $(25$ and $47 \mathrm{~mm}$ diameter) and limiting the filtration to aliquots of the sample reduced the problem. Visualization was also greatly improved when staining was eliminated from the procedure and subsequent counts were done under phase contrast lenses.

These studies have shown that the micropore filtration technique can be a useful tool for the routine estimation of sperm concentrations in semen samples and for the determination of sperm distribution in the female reproductive tract. Further studies, however, are required to establish more firmly the relative accuracy of this method and the potential application of micropore filtration methods to reproductive problems requiring the separation of spermatozoa from their fluid environment.

I would especially like to thank Mrs Marguerite J. Mueller for technical assistance in these studies.

\section{REFERENCES}

Austin, G. R. (1948) Number of sperms required for fertilization. Nature, Lond. 162, 534.

Braden, A. W. H. (1953) Distribution of sperms in the genital tract of the female rabbit after coitus. Aust. F. biol. Sci. 6, 693.

Chang, M. C. (1951) Fertilization in relation to the number of spermatozoa in the fallopian tubes of rabbits. Annali Ostet. Ginec. 73, 918.

Glover, F. A. \& Phipps, L. W. (1962) Preliminary study of an electronic method of counting and sizing bull spermatozoa. 7. Reprod. Fert. 4, 189.

WiLlet, E. L. \& BuCKNER, P. J. (1951) The determination of numbers of spermatozoa in bull semen by measurement of light transmission. J. Anim. Sci. 10, 219. 